Research Article

\title{
In Vitro Comparison of the Internal Ribosomal Entry Site Activity from Rodent Hepacivirus and Pegivirus and Construction of Pseudoparticles
}

\author{
Stuart Sims $\mathbb{D}^{1},{ }^{1}$ Kevin Michaelsen, ${ }^{1}$ Sara Burkhard, ${ }^{2}$ and Cornel Fraefel ${ }^{1}$ \\ ${ }^{1}$ Institute of Virology, University of Zurich, Zurich, Switzerland \\ ${ }^{2}$ Department of Infectious Diseases, University Hospital of Zurich, Zurich, Switzerland \\ Correspondence should be addressed to Stuart Sims; stuart.sims@hotmail.com
}

Received 4 February 2021; Accepted 20 July 2021; Published 30 July 2021

Academic Editor: Jay C. Brown

Copyright (c) 2021 Stuart Sims et al. This is an open access article distributed under the Creative Commons Attribution License, which permits unrestricted use, distribution, and reproduction in any medium, provided the original work is properly cited.

The $5^{\prime}$ untranslated region ( $5^{\prime}$ UTR) of rodent hepacivirus (RHV) and pegivirus (RPgV) contains sequence homology to the HCV type III internal ribosome entry sites (IRES). Utilizing a monocistronic expression vector with an RNA polymerase I promoter to drive transcription, we show cell-specific IRES translation and regions within the IRES required for full functionality. Focusing on RHV, we further pseudotyped lentivirus with RHV and showed cell surface expression of the envelope proteins and transduction of murine hepatocytes and we then constructed full-length RHV and RPgV replicons with reporter genes. Using the replicon system, we show that the RHV NS3-4A protease cleaves a mitochondrial antiviral signaling protein reporter. However, liverderived cells did not readily support the complete viral life cycle.

\section{Introduction}

The hepatitis $\mathrm{C}$ virus (HCV) infects more than 71 million people worldwide [1], which can lead to liver failure and hepatocellular carcinoma and presents a major global health burden. While the introduction of new direct-acting antiviral drugs (DAAs) has improved treatment response rates and heralded a new era of HCV treatment [2], the cost and availability of DAAs along with drug resistance and chronic/ nonreversible liver damage due to late onset of symptoms and delayed treatment initiation after HCV infection remain an issue [3]. A small animal model for HCV would allow for the testing of vaccines which could potentially solve these problems.

HCV was discovered in humans 20 years ago [4] but for a long time, researchers failed to identify an animal viral homologue. This all changed with the use of high throughput deep sequencing, which has shed light on the evolutionary origins of the virus. The first HCV homologue was discovered in 2011 in canines [5] and was quickly followed by the discovery of homologues in rodents $[6,7]$, equine $[8,9]$, primates [10], bovine $[11,12]$, and then, the first nonmammalian species, sharks [13].

The rodent HCV homologues were termed rodent hepaciviruses (RHV) and were first identified in deer mice (Peromyscus maniculatus), a species known to carry hantavirus, desert woodrats (Neotoma lepida), and hispid pocket mice (Chaetodipus hispidus) [7]. The same year RHV was described in European bank voles (Myodes glareolus) and South African four-striped mice (Rhabdomys pumilio) [6]. This was succeeded by the discovery of an RHV from Norway rats (Rattus norvegicus) in New York City [14].

The subsequently discovered RHV (RHV-rn1) from Norway rats was used to make a small animal model for hepaciviral infection, utilising a reverse genetics approach. In this system, the researchers showed its hepatotropic replication in inbred and outbred rat strains [15]. Emulating $\mathrm{HCV}$ infection, they also showed that persistent infection leads to gradual liver damage and that the HCV antiviral drug sofosbuvir suppresses replication of RHV-rn1. This model can be used to study the mechanisms of HCV persistence, immunity, and pathogenesis. 
Though rats are the usual hosts of RHV-rn1, it has also been shown that the virus is capable of establishing a persistent infection in immunocompromised mice lacking type I interferon and adaptive immunity [16]. However, immunocompetent mice clear the virus in a few weeks. Because this mouse model only results in an acute infection, a fully immunocompetent mouse model in which a chronic infection and downstream liver damage can be established is still in need. Additionally, the availability of knockout mice would aid the study of the pathogenesis of HCV related liver damage.

While these studies could lead the way to future vaccines, they also present the possibility of zoonotic sources of $\mathrm{HCV}$ infection in humans [17]. The genomes of RHV encode for a polyprotein that is predicted to be cleaved into 10 proteins, as with $\mathrm{HCV}$, but shows a $66-77 \%$ amino acid divergence from HCV in the structural genes [7, 18]; therefore, tropism and pathogenicity may also differ between the viruses. In particular, the $5^{\prime}$ untranslated regions (UTRs) of RHV are highly divergent from the corresponding regions of $\mathrm{HCV}$, and there is a large difference between the different rodent clades (RHV, RHV1, RHV2, RHV3, and RHV-rn1).

The high-level expression of mir-122 in liver cells allows $\mathrm{HCV}$, containing two mir-122 target sequences in the $5^{\prime} \mathrm{UTR}$, to replicate and is one reason for HCV hepatotropism [19]. Similarly, the RHV (NC_021153) found in deer mice contains one such mir-122 target sequence in its $5^{\prime} \mathrm{UTR}$ [7] suggesting liver cell specificity and further has a $200 \mathrm{nt}$ sequence with no homology to other hepaciviruses $5^{\prime}$ UTRs. The RHV-1 (KC411777)) 5'UTR contains structural elements typical of both pegi- and HCV-like IRES and contains one mir-122 target region, while RHV-1 and RHV-2 (KC411784) are identical in structure and only contain a few nucleotide exchanges, with RHV-3 (KC411807) and RHVrn1 being more similar to typical HCV-like IRES structures $[6,15]$ (Figure 1).

Along with RHV, a rodent pegivirus (RPgV) was also discovered in white-throated wood rats (Neotoma albigula) [7]. Pegiviruses are a new genus of the family of the Flaviviridae, encompassing the human GBV-A, GBV-C/HGV/ $\mathrm{HPgV}-1, \mathrm{GBV}-\mathrm{D}$ and $\mathrm{HPgV}-2$ viruses. These pegiviruses are considered to be nonpathogenic and in the case of $\mathrm{HPgV}$ has even been reported to be beneficial in coinfections with HIV or Ebola [20-22]. However, two pegiviruses were discovered in equine. The first, Theiler's disease-associated virus (TDAV), is suspected to be the causative agent for an outbreak of acute hepatic disease occurring on a horse farm [23]. The second, equine pegivirus $(\mathrm{EPgV})$, like the human pegiviruses, is considered to be nonpathogenic [24].

The viral RNA structures play important roles in both translation and replication. Specifically, the $5^{\prime} U T R$ containing the IRES promotes the initiation of protein synthesis in a cap-independent manner. IRES's are diverse in sequence and structure and these differences contribute to tropism; the focus of this study is to assess how these differences in the $5^{\prime}$ UTRs of RHV and RPgV affect translation and to establish a reverse genetics system utilizing the RNA polymerase I promoter and terminator in a DNA transfection system $[25,26]$ to allow for quick and easy mutation and screening.

\section{Materials and Methods}

Cells. Hepa1-6 (ATCC ${ }^{\circledR}$ CRL-1830 ${ }^{\mathrm{TM}}$ ), MEFs IRF3 ${ }^{-/-}$, NIH 3T3 (ATCC ${ }^{\circledR}$ CRL-1658 ${ }^{\mathrm{TM}}$ ), BHK-21 (ATCC $^{\circledR}$ CCL-10 ${ }^{\mathrm{TM}}$ ), HEK 293T (ATCC ${ }^{\circledR}$ CRL-11268 ${ }^{\mathrm{TM}}$ ), Huh7.5-RFP-MAVS from Dr Charles M Rice, HepG2 (ATCC ${ }^{\circledR} \mathrm{HB}-8065^{\mathrm{TM}}$ ) and Vero cells (ATCC ${ }^{\circledR}$ CCL$81^{\mathrm{TM}}$ ) were cultured in DMEM (Sigma-Aldrich), 10\% FBS and $1 \%$ penicillin-streptomycin.

Plasmid Construction. Monocistronic reporter plasmids containing viral 5'UTR were constructed in pUC19. First, pUC19 was digested with EcoR1 and HIndIII (NEB), and then a minimal RNA polymerase I (RNA Pol I) promoter and terminator (synthesised by Twist Biosciences) was inserted into the digested plasmid by In-Fusion cloning (Takara Bio); this plasmid was named pOLI (Figure S1A).

The plasmid pOLI was linearized with PpuMI. Viral 5' and $3^{\prime \prime}$ UTR (synthesised by Twist Biosciences) (HCV IRES taken from pFR_HCV_xb (Addgene)) along mCitrine were cloned into the linearized pOLI by InFusion cloning (Takara Bio) (Figure S1B). Deletions and additions to the monocistronic reporter plasmids were created by PCR and In-Fusion cloning (Takara Bio).

Plasmids containing viral structural genes were constructed in pUC19 by In-Fusion cloning (Takara Bio) using hepacivirus CE1E2 region (synthesised by Twist Biosciences), along with a CMV promoter and $\mathrm{BGH}$ polyA (Figure S1C). Flag tag and c-Myc tag sequences were inserted into this plasmid by PCR and In-Fusion cloning (Takara Bio); this plasmid was named $\mathrm{pC}^{\mathrm{My}-}$ ${ }^{\mathrm{c}} \mathrm{E} 1 \mathrm{E} 22^{\mathrm{Flag}}$. The capsid gene was deleted from $\mathrm{pC}^{\mathrm{My}-}$ ${ }^{\mathrm{c}} \mathrm{E} 1 \mathrm{E} 2^{\text {Flag }}$ by PCR and In-Fusion cloning (Takara Bio) resulting in the plasmid $\mathrm{pE} 1 \mathrm{E} 2^{\text {Flag }}$.

To construct plasmids containing full-length RHV1 and $\mathrm{RPgV}$ viral genomes, the respective monocistronic vector (pOLI.IRES.Cirtine) was PCR linearized, and gene fragments, $1.5-2 \mathrm{~kb}$ (synthesised by Twist Biosciences), covering the full-length viral coding region were then cloned in by In-Fusion cloning (Takara Bio) replacing mCitrine. The plasmids were named pOLI.RHV1 and pOLI.RPGV (Figure S1D).

For constructing a reporter plasmid, mScarlet-BSD (synthesised by Twist Biosciences) was cloned into the plasmid containing the full-length virus in-between NS5A and NS5B, while also duplicating the cleavage sequence by In-Fusion cloning (Takara Bio). These plasmids were named pOLI.RHV1.SB and pOLI.RPgV.SB (Figure S1E).

Transfection. All transfections were carried out using Lipofectamine ${ }^{\circledR}$ LTX with Plus ${ }^{\mathrm{TM}}$ Reagent (Thermo Fisher Scientific) in Opti-MEM (Gibco) according to the manufacturer's specifications. 

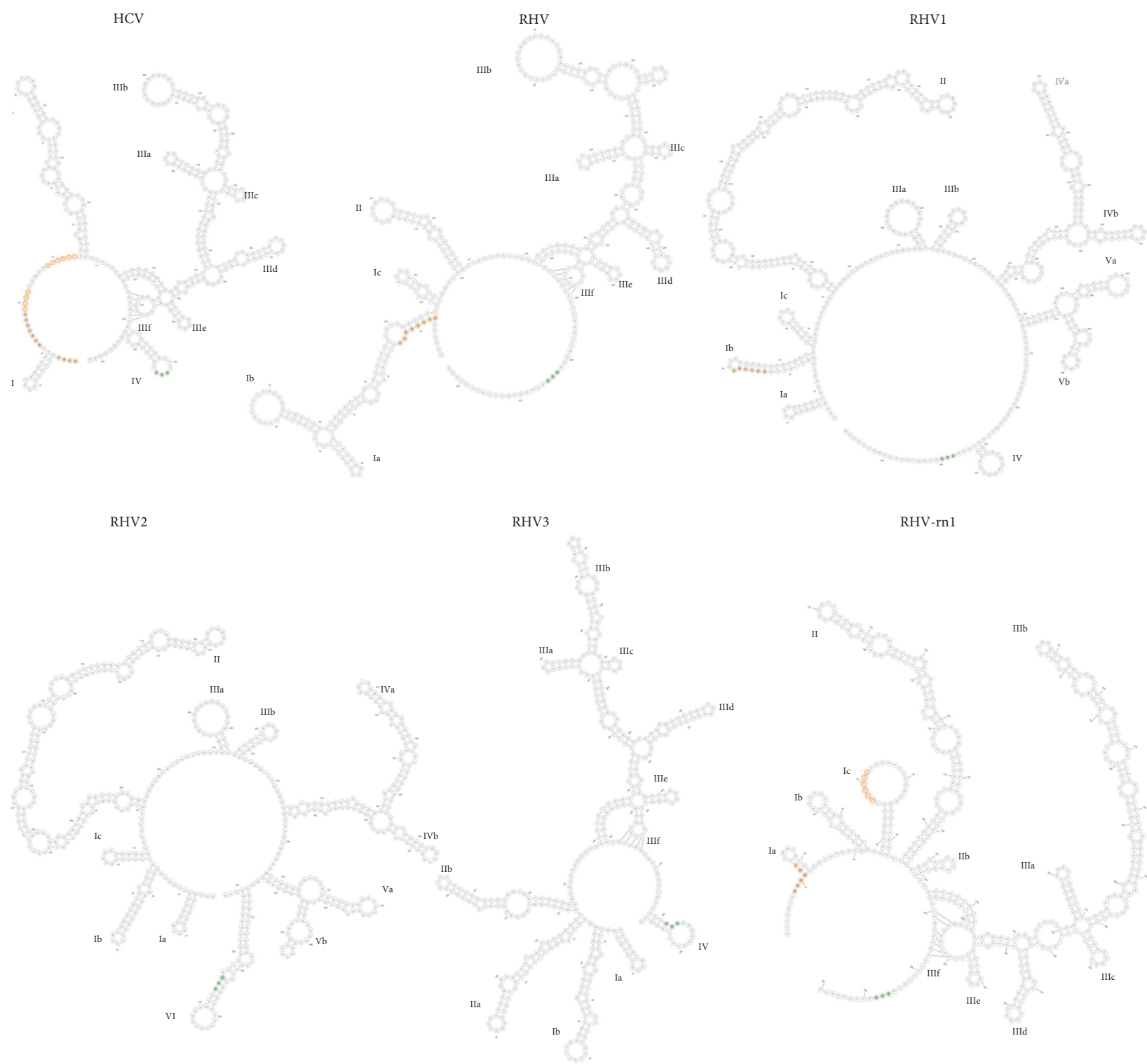

RHV-rn1

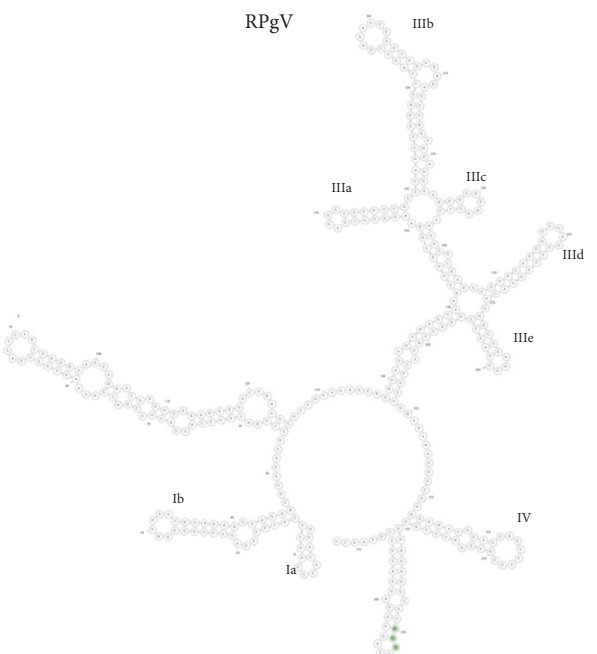

Figure 1: Predicted RNA secondary structures within HCV, RHV, RHV1, RHV2, RHV3, RHV-rn1, and RPgV 5' UTR. The sequence corresponding to the mir-122 binding sites within the $5^{\prime}$ UTR is highlighted in orange; the first binding sites are represented by filled circles and the second by the outline. HCV contains two mir-122 binding sites and RHV, RHV1, and RHV-rn1 contain one predicted binding site. RHV2/3 and $\mathrm{RPgV}$ do not contain a mir-122 binding site. The nucleotides corresponding to the AUG start codon are highlighted by filled green circles. 
Western Blot. Cells were lysed in RIPA buffer $(150 \mathrm{~mm}$ $\mathrm{NaCl}, 50$ mm Tris/HCl pH 7.6, 1\% Nonidet P40, 0.5\% sodium deoxycholate, and $5 \mathrm{~mm}$ EDTA) supplemented with cOmplete ${ }^{\mathrm{TM}}$ Protease Inhibitor Cocktail (Roche) for 15 min on ice. The lysate was run on a $12 \%$ SDSPage and transferred to PVDF membrane (Bio-Rad). Membranes were incubated for 1 hour in PBS with 5\% nonfat dry milk and then stained with primary and subsequently secondary antibodies for 1 hour in PBS with $1 \%$ nonfat dry milk. Immunocomplexes were detected using an Odyssey ${ }^{\circledR}$ Fc Imaging System (LICOR Biosciences).

Intracellular Immunofluorescence. Cells were grown on glass coverslips, washed with PBS prior to fixation in formaldehyde $(3.7 \% w / v$ in $\mathrm{PBS})$ for $10 \mathrm{~min}$ at room temperature, permeabilized for $5 \mathrm{~min}$ with $0.1 \%$ Triton $\mathrm{X}-100$ in PBS, and blocked with 3\% BSA in PBS for $30 \mathrm{~min}$. Primary and secondary antibodies were diluted in PBS containing $1 \%$ BSA, and cells were stained with primary antibody for 1 hour, washed $3 \mathrm{x}$ with PBS, and then stained with secondary antibody for 1 hour, followed by staining with $0.1 \mu \mathrm{g} / \mathrm{ml}$ DAPI in PBS for $5 \mathrm{~min}$ and application of Prolong Gold Antifade reagent (Invitrogen).

Extracellular. Cells were grown on glass coverslips, incubated with PBS containing 4\% FBS (Fetal Bovine Serum) for $30 \mathrm{~min}$. Primary and secondary antibodies were diluted in PBS containing 4\% FBS, cells were stained with primary antibody for 1 hour, washed $3 \mathrm{x}$ with PBS, and then stained with secondary antibody for 1 hour. Cells were then stained with Wheat Germ Agglutinin, Alexa Fluor ${ }^{\mathrm{TM}} 594$ conjugate as per manufacturers protocol. Cells were fixed in formaldehyde $(3.7 \% \mathrm{w} / \mathrm{v}$ in $\mathrm{PBS})$ for $10 \mathrm{~min}$ at room temperature, permeabilized for 5 min with $0.1 \%$ Triton X-100 in PBS, and stained with $0.1 \mu \mathrm{g} / \mathrm{ml}$ DAPI in PBS for $5 \mathrm{~min}$ followed by application of Prolong Gold Antifade reagent (Invitrogen).

Images were acquired using a confocal microscope (Leica specify type) and Z-stacks and analysed with ImageJ.

Antibodies. Rat anti-DYKDDDDK (clone L5, Biolegend), mouse anti-c-myc (clone 9E11, Biolegend), mouse anti- $\beta$-actin (clone 2F1-1, Biolegend), mouse J2 anti-dsRNA IgG2a (Sciscons), goat anti-rat IgG $(\mathrm{H}+\mathrm{L})$ Alexa Fluor 488 (Invitrogen), IRDye ${ }^{\circledR} 800 \mathrm{CW}$ goat anti-rat IgG, and IRDye ${ }^{\circledR}$ 680RD donkey anti-mouse IgG (LI-COR Biosciences).

Flow Cytometry. Single cell suspensions were generated and kept in FACS buffer (2\% FCS, 5 mm EDTA in PBS). Cells were analysed using a Gallios flow cytometer (Beckman Coulter) and FlowJo software and gated on viable cells using the live/dead fixable near-IR dead cell stain kit (Invitrogen).

Lentivirus. Plates were seeded with HEK 293T in DMEM and 3\% FCS and then transfected with pLKOgfp (Addgene), pCMV $\triangle$ R8.2 (Addgene), and either
pMD2.G (Addgene) or pE1E2 at a ratio of 1:1:0.1, respectively, using lipofectamine LTK (Thermo Fisher Scientific) and Opti-mem (Gibco). At 6 hours after transfection, media were replaced and $72 \mathrm{~h}$ after transfection, supernatant was harvested and passed through a $0.45 \mu \mathrm{m}$ filter.

2.1. RNA Structure Prediction. The RNA sequences secondary structure was predicted with RNA fold and visualization was performed using force directed graph layout (Forna); both are hosted on ViennaRNA Web services (http://rna.tbi.univie.ac.at/forna/).

2.2. RNA Extraction and cDNA Generation. Cells from 24well plate were washed in PBS and resuspended in Trizol (Sigma-Aldrich, USA), and RNA was isolated by isopropanol precipitation, washed with $70 \%$ ethanol, and resuspended in DEPC-water. RNA was DNAse treated (Invitrogen, Paisley, UK) and subjected to RT-PCR using 100 ng purified RNA. For RT-PCR, the high-capacity cDNA archive Kit from Applied Biosystem (ABI PRISM, Warrington, United Kingdom) was used according to the specifications of the manufacturer.

2.3. Quantitative Real-Time PCR for Selected Genes. Quantitative real-time PCR was performed using a Light cycler 480 Real-Time PCR System (Roche Diagnostics) and the LightCycler 480 probes master reaction mix (Roche Diagnostics) following the manufacturer's protocol. Data analysis was performed with LightCycler 480 Software (Roche Diagnostics). Oligonucleotides sequences used as primers for quantitative real-time PCR and corresponding probes were designed according to the guidance of the Universal Probe library from Roche applied Science. Thermal cycling started with HotStarTaq activation during $10 \mathrm{~min}$ at $95^{\circ} \mathrm{C}$. Thereafter, 45 cycles of amplification were run consisting of $10 \mathrm{~s}$ at $95^{\circ} \mathrm{C}, 30 \mathrm{~s} 60^{\circ} \mathrm{C}$, and $20 \mathrm{~s}$ of $72^{\circ} \mathrm{C}$. A negative control containing reagents only and serial dilutions of cDNA was included in each run. Each sample was measured as a triplicate and the average concentration was used. For LightCycler analysis, expression of hypoxanthine phosphoribosyltransferase gene (HPRT) was used for normalization. Relative expression of samples was calculated by the comparative cycling threshold method $(\Delta \Delta \mathrm{CT})$ and then setting the samples transfected with HCV IRES as the benchmark.

GeneBank Accession Numbers. RHV (Hepacivirus E), NC_021153; RHV1 (Hepacivirus J), KC411777; RHV2 (Hepacivirus F), KC411784; RHV3 (Hepacivirus I), KC411807; RHV-rn1 (Hepacivirus G), KX905133.1; RPgV, NC_021154.

\section{Results}

3.1. RHV and RPgV IRESs Are Functional in Rodent Cells. To test viral IRES driven translation, a monocistronic plasmid vector was constructed containing a minimal RNA 
polymerase I promoter in front of the full-length viral $5^{\prime}$ UTR followed by a fluorescent maker, the viral $3^{\prime}$ UTR, and the RNA polymerase I terminator (Figure $2(\mathrm{a})$ ). The $\mathrm{HCV}$ IRES was used as a positive control along with a control plasmid containing a scrambled sequence in place of the viral 5' UTR. We constructed plasmids containing RHV, RHV1, RHV2, RHV3, RHV-rn1, and RPgV 5' UTR from previously published sequences.

These plasmids were transfected into the murine hepatocyte cell line Hepa1-6, with the HCV and RPgV IRES driving the highest level of translation at 72 hours after transfection with a mean fluorescence intensity (MFI) of 30, followed by RHV1 and RHV2 with an MFI of 22 and 14, respectively. RHV3 and RHV-rn1 IRES drove translation at a level only slightly above background and RHV was not functional in Hepa1-6 (Figure 2(b)). To further assess the level of RNA transcripts of mCitrine within the cells, we preformed qRTPCR on transfected cells. There was no statistical difference in the level of transcripts between HCV, RHV, RHV1, RHV2, RHV3, RHV-rn1, RPgV, and the control plasmid containing a scrambled sequence in place of the viral $5^{\prime} \mathrm{UTR}$. The other control plasmid contains RHV1 5'UTR but no Pol I promoter did yield a readily detectable level of RNA (Figure S2).

To further assess IRES function in murine cells, we tested two murine embryonic fibroblasts cell lines, MEFs and NIH 3T3. Transfection of MEFs with the plasmids revealed that RHV1 drove the highest level of translation at an MFI of 9 followed by RHV2 at an MFI of 5 (Figure 3(c)). HCV, RHV, RHv3, RHV-rn1, and RPgV generated signals only slightly above the negative control. In NIH3T3, the RPgV drove the highest level of translation with an MFI of 17 followed by $\mathrm{HCV}$ with an MFI of 7; again, RH1 and RHV2 were functional but at low levels, and RHV, RHV3, and RHV-rn1 were not functional (Figure 3(d)).

The viruses originate from different rodent species; therefore, the baby hamster kidney cell line was tested to assess if the IRESs are functional in this cell line. As with the previous cell lines, the RPgV IRES drove high levels of translation; also the RHV1 and RHV2 were capable of driving high levels followed by HCV; again, the RHV, RHV3, and RHV-rn1 were not functional (Figure 2(e)).

\subsection{RHV and RPgV IRESs Show Differing Functions in Human} Cell Lines. In the human hepatocyte cell line, Huh7.5 which expresses high levels of mir122, and the RPgV IRES drives the highest levels of translation followed by HCV and RHV2. The RHV-rn1 drove low levels of translation and, as with murine cells, RHV and RHV3 did not yield any signal (Figure 2(f)). Another human hepatocyte cell line, HepG2 that does not express mir122, was also used to test the IRES's function: in these cells, HCV drove the highest levels followed by RPgV and RHV2, while the expressions from RHV, RHV1, RHV3, or RHV-rn1 were at background level (Figure 2(g)).

To investigate if the IRESs are functional in nonhepatocyte cells lines, we used HEK 293T and Vero cell lines. In HEK 293T, the HCV IRES showed the highest level of translation followed by the RPgV IRES and RHV2, while that of RHV, RHV1, RHV3, and RHV-rn1 was at the lowest level (Figure 2(h)). In Vero cells, the RPgV produced high levels of translation, over twice that of HCV; RHV2 was the only other IRES that was functional in these cells although at a very low level when compared to RPgV (Figure 2(i)).

\subsection{Deletions Abrogate the Function of RHV1 and RPgV IRES.} To further assess the structural requirements of RHV1 IRES for full functionality, we made several different constructs. The first contained an additional 20 nucleotides of virus sequences downstream of the start codon. When transfected into Hepa1-6 cells, this construct did not lead to a difference in the levels of translation in comparison with the construct containing just the $5^{\prime}$ UTR. Two deletion constructs were made; in RHV $\Delta \mathrm{I}$, the $5^{\prime}$ three stem loops $(\mathrm{Ia} / \mathrm{b} / \mathrm{c})$ were deleted (Figure 3(a)). This deletion decreased the IRES function by $90 \%$. The $\mathrm{Va}$ and $\mathrm{Vb}$ stem loops were deleted from RHV $1 \Delta \mathrm{II}$ and again led to a decrease in function by $90 \%$ in the murine hepatocyte cell line Hepa1-6 (Figure 3(c)).

For the RPgV IRES, we also added an additional 20 nucleotides of virus sequences downstream of the start codon. This again had no effect on the levels of translation in comparison to the construct containing just the $5^{\prime}$ UTR. To further assess the sequence required for driving translation, three constructs were made with deletions, RPgV $\Delta \mathrm{I}$ and RPgV $\Delta$ II have deletions to the $5^{\prime}$ of the IRES with RPgV $\Delta \mathrm{I}$ having the first two stem loops (Ia/b) and RPgV $\Delta$ II three stem loops deleted (Ia/b and II) (Figure 3(b)). RPgV $\Delta$ III has two internal stem loops deleted (IIId/e). The $5^{\prime}$ deletions to RPGV had no effect on the levels of translation. However, the internal deletions in $\mathrm{RPgV} \Delta \mathrm{III}$ led to a reduction in translation of $53 \%$ (Figure 3(d)).

3.4. Expression of E1E2. Previous studies examining the subcellular localization of HCV E1 and E2 used cells transfected with a plasmid expressing the $\mathrm{E} 1$ and $\mathrm{E} 2$ proteins [27]. These studies concluded that the HCV structural proteins are expressed on the cell surface, based on immunofluorescence detection. In order to examine the localization of RHV structural proteins in an expression system, we cloned the structural region (capsid-E1-E2) of RHV into a plasmid containing the CMV promoter; we then added the $\mathrm{c}-\mathrm{Myc}$ tag to the $5^{\prime}$ end of the capsid protein and the Flag tag to the $5^{\prime}$ end of the E2 protein following the E1E2 cleavage sequence (Figure 4(a)).

HEK 293T cells were transfected with the vectors $\mathrm{pE} 1 \mathrm{E} 2^{\text {Flag }}$ or $\mathrm{pC}^{\mathrm{Myc}} \mathrm{E} 1 \mathrm{E} 2^{\text {Flag }}$ and after 48 hours lysed for SDS-PAGE. The E2 protein was detected in cells transfected with either expression vector using an anti-Flag tag antibody (Figure 4(a)), and the capsid was detected in cells transfected with the $\mathrm{pC}^{\mathrm{Myc}} \mathrm{E} 1 \mathrm{E} 2^{\text {Flag }}$ expression vector using an anti-cMyc antibody. The proteins detected were of the predicted size, showing that posttranslational cleavage was complete.

In order to determine if the RHV glycoproteins expressed from these vectors also exhibit an intracellular colocalization, we examined transfected cells by immunofluorescence for capsid and E2 expression; both were shown to colocalize (Figure 4(b)). We also assessed if the envelope 


\begin{tabular}{|l|l|l|l|l|}
\hline PollP & 5'UTR & mCitrine & $3^{\prime}$ UTR & PollT \\
\hline
\end{tabular}
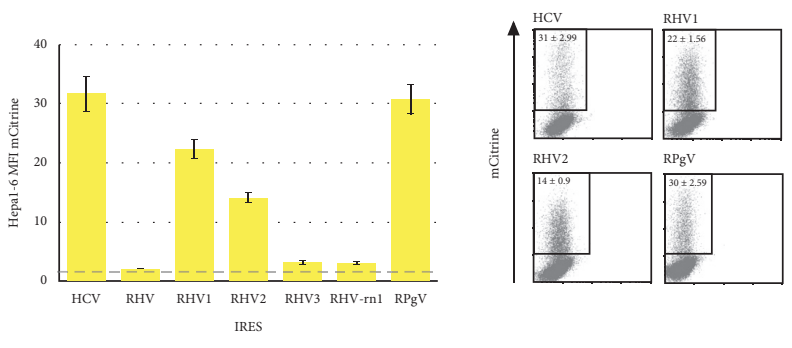

(b)
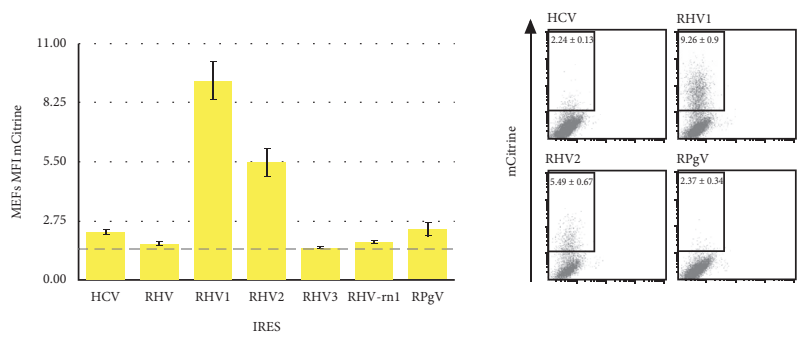

(c)
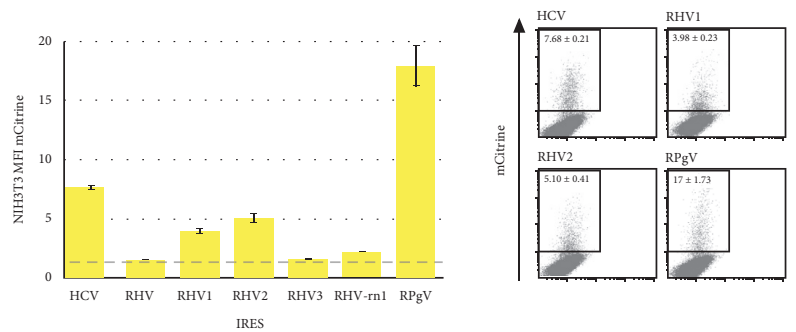

(d)

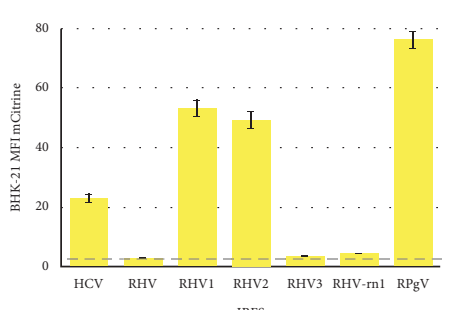

IRES

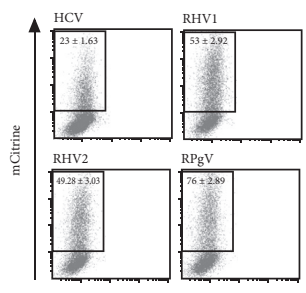

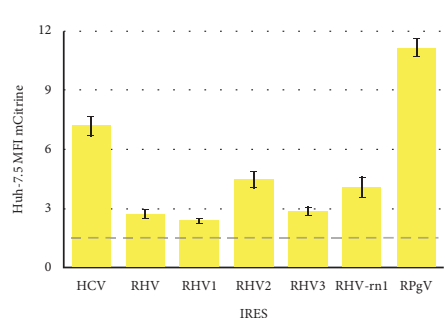
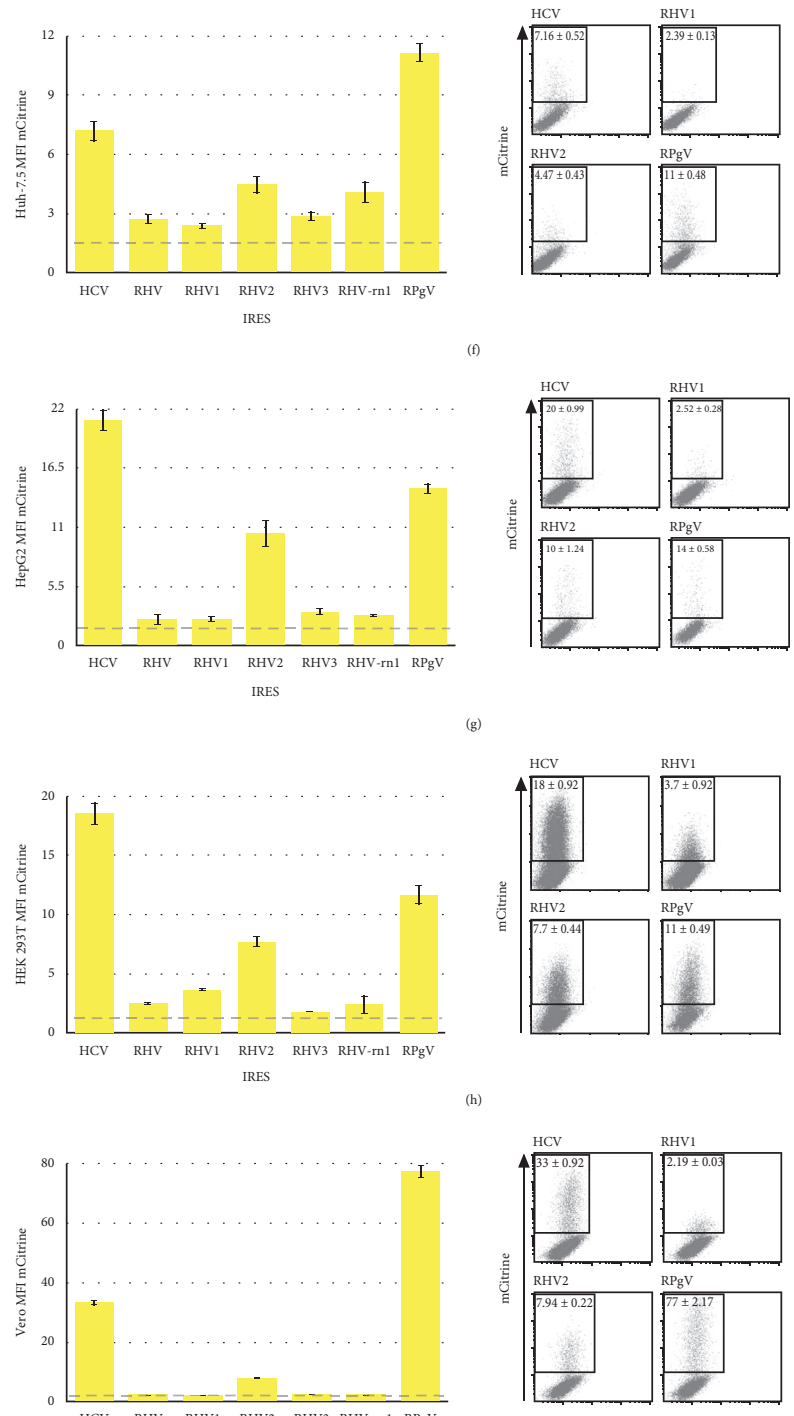

FIGURE 2: RHV and RPGV IRES activity in different cell types. Schematic of the monocistronic vectors used (a). Hepa1-6 (b), MEFs (c), NIH 3T3 (d), BHK-21 (e), Huh7.5 (f), HEK 293T (h), and Vero (i) cells were transfected with the indicated plasmids. Cells were harvested and analysed by flow cytometry at 48 h.p.t.; bar graphs show MFI for mCitrine ( $n \geq 8$, mean \pm SEM of at least three independent experiments), and representative FACS plots on the right of each graph numbers represent the MFI (mean \pm SEM). The dashed line represents the fluorescent output of the control plasmid containing a scrambled sequence in place of the viral $5^{\prime}$ UTR.

protein was expressed on the cell surface. Staining with antiflag antibody to detect E2 indeed showed punctate staining on the cell membrane and when combined with wheat germ agglutinin to stain the cell membrane, it showed colocalization with the flag antibody (Figure 4(c)). This indicates that a proportion of E2 is surface-expressed.

To determine if the surface-localized E1E2 could mediate viral entry, we produced a GFP encoding lentivirus vector pseudotyped with the RHV, RHV1, RHV2, and RHV3 E1E2 proteins by transfecting HEK 293T cells with pE1E2 and the lentivirus backbone and packaging plasmid, and $72 \mathrm{~h}$ later harvesting and filtering the supernatant. We then tested if the
E1E2-pseudotyped lentivirus vectors were entry-competent. Supernatants from the cotransfected cells were applied to Hepa1-6 cells and GFP reporter expression assayed $72 \mathrm{~h}$ later. RHV1 E1E2-pseudotyped lentivirus vectors gave rise to a small number of GFP positive cells, when compared to VSVG pseudotyped lentivirus. Lentivirus vectors pseudotyped with RHV, RHV2, and RHV3 or lacking envelope glycoprotein failed to give rise to any GFP positive cells (Figure 4(d)). This indicates that only RHV1 pseudotyped lentivirus vectors can mediate viral entry in Hepa1-6 cells resulting in reporter gene expression. This data also indicates that surface-expressed RHV1 E1E2 heterodimers are functional. 


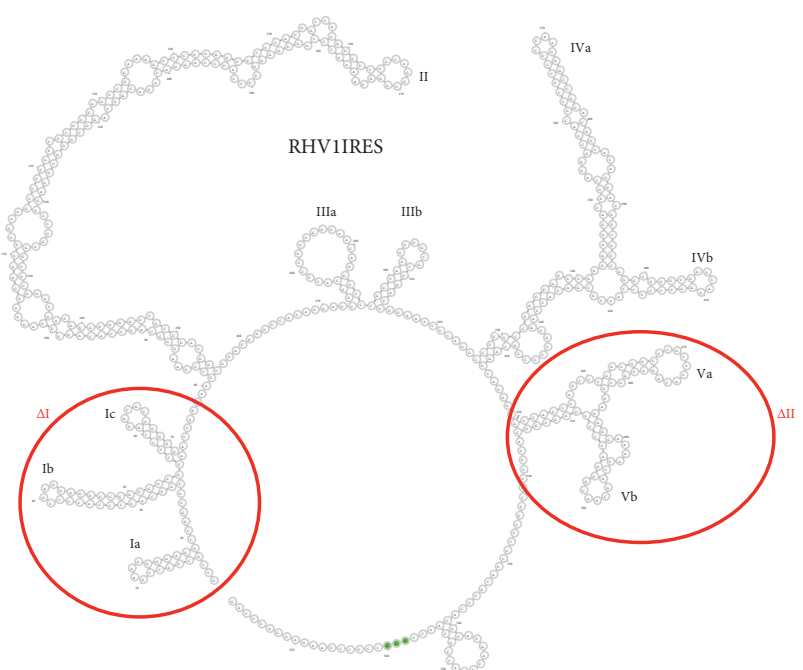

(a)

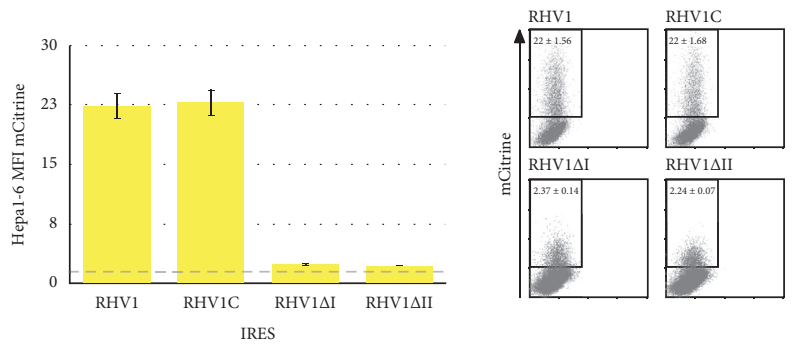

(c)

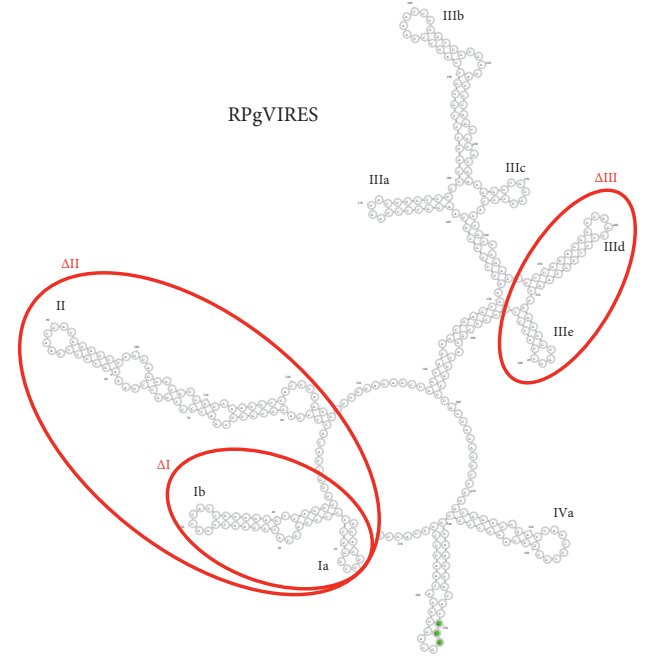

(b)
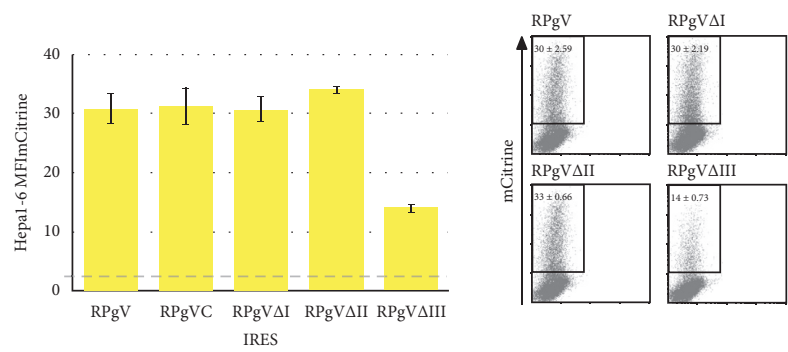

(d)

FIgURE 3: Deletions to RHV1 and RPgV 5' UTR abrogate IRES translation. Predicted RNA secondary structure of RHV1 (a) and RPgV (b). Red circles indicate areas deleted from plasmids. Hepa1-6 cells were transfected with RHV1 (c) and RPgV (d) plasmids. Cells were harvested and analysed by flow cytometry at 48 h.p.t. Bar graphs show MFI for mCitrine ( $n \geq 8$, mean \pm SEM of at least three independent experiments) and representative FACS plots on the right of each graph; numbers represent the MFI (mean \pm SEM). The dashed line represents the fluorescent output of the control plasmid containing a scrambled sequence in place of the viral $5^{\prime}$ UTR.

\section{Discussion}

The five rodent hepacivirus IRESs we tested showed different levels of ability to drive translation using in a monocistronic vector across varying cell lines. While the use of a monocistronic vector, utilizing RNA polymerase I, avoids the potential of readthrough in comparison to bicistronic vectors; its drawback is that we were not able to directly compare expression levels between cell types due to their difference in susceptibility to transfection. However, the RHV, RHV3, and RHV-rn1 IRESs were either not functional or drove expression at very low levels. This comes as a surprise as the RHV-rn1 virus has already been shown to replicate in both mice and rats.

Both RHV1 and RHV2 drive high levels of translation in murine hepatocytes, MEFs and BHK-21 cells. In human hepatocytes, the RHV2 IRES outperformed RHV1 which is of interest as they are similar in sequence and therefore are likely to have a similar structure. In the case of RHV1, deleting the predicted initial three stem loops abrogates IRES function, suggesting that the full $5^{\prime}$ UTR sequence is required to maintain high levels of expression. Also, unlike HCV, where previous studies have shown that the inclusion of 12-30 nt of the core protein coding sequence was essential for an efficient IRES activity [28], additional nucleotides from the core protein of RHV1 did not increase transcription levels.

The RPgV 5'UTR has little significant similarity with any known pegivirus but drives high levels of expression in all cell types tested. By deleting specific regions, we were able to show that the initial 126 nt of the $5^{\prime}$ UTR does not contribute to IRES function and that stem loops IIId/e are essential for maintaining high levels of expression. It would be of significance in the future to confirm the predicted structures of RPgV and RHV1 IRES's potentially using RNA SHAPE.

RHV1 structural genes (C, E1, and E2) expressed from plasmid were shown to be cleaved and yielded proteins of the correct size. Moreover, RHV1 E1E2 supported transduction of hepatocytes when used to pseudotype lentivirus vectors. Further studies will need to be carried out to find the specific entry receptors, initially blocking CD 81 and HCV entry receptors and testing susceptibility of transduction in alternative cell lines, but this initial experiment hints at the hepatotropic potential of RHV1 in mice.

The generation of viral pseudotypes is one of the most widely used methods for assaying functional receptors, allowing attachment, penetration, and uncoating to be 


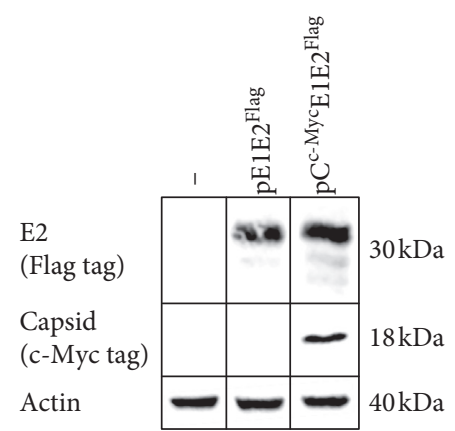

(a)

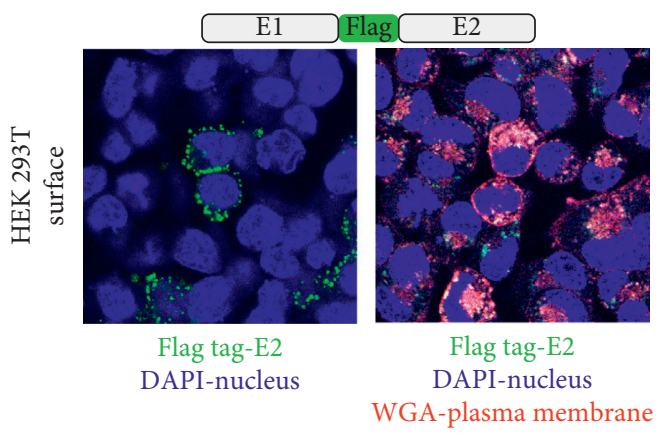

(c)

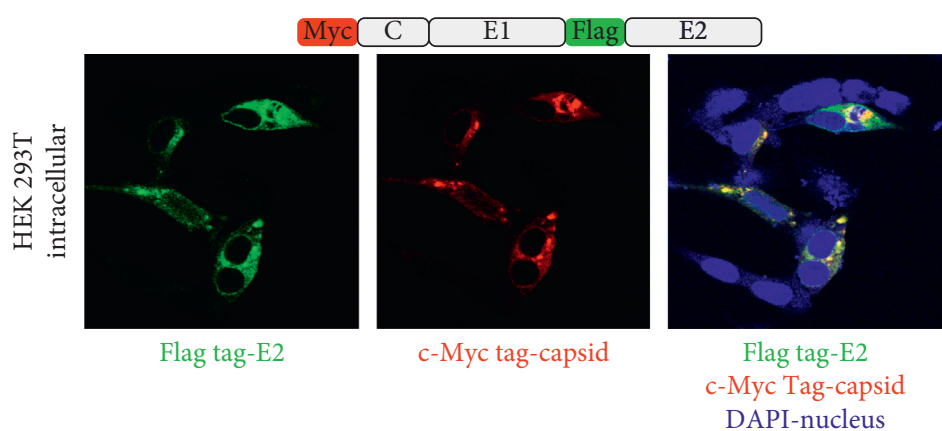

(b)

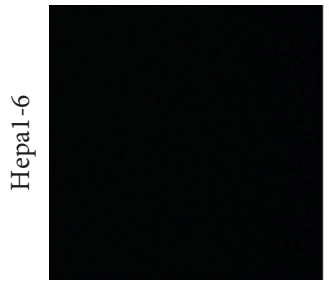

RHV

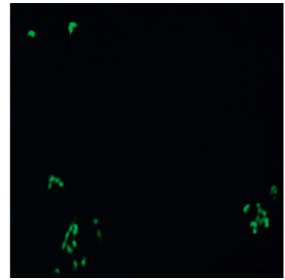

RHV1

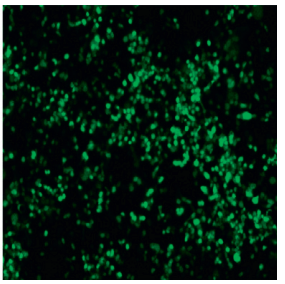

VSVG (d)

FiguRE 4: Expression of RHV envelope proteins. Schematic of the RHV1 envelope expression vector and western blot of cell lysate at 48 h.p.t. from transfected HEK 293T (a) Intracellular immunofluorescence of 293T cells at 48 h.p.t. with RHV1 envelope expression vector, stained with flag tag in green, c-Myc tag in red, and combined with DAPI stain (b) Extracellular immunofluorescence for E2 by flag tag stain (green) and combined with WGA (red) and DAPI (blue) from 293T at 48 h.p.t. with RHV1 envelope expression vector, and images represent one slice from z-stack (c) GFP-lentivirus vector pseudotyped with RHV or RHV1 envelope proteins or VSVG were incubated on Hepa1-6 cells; GFP positive cells indicate transduction (d).

studied. This study lays the groundwork for using RHV1 pseudotype particles to be used to asses these important parts for the viral replication cycle and could also be used to study cell tropism in a murine model and the antigenicity of the functional E1 and E2 glycoproteins.

Our efforts to make an RHV and $\mathrm{RPgV}$ replication competent model in vitro have so far proved unfruitful. Using full-length viral constructs, we tested the human hepatoma cell line (Huh-7.5) containing a MAVS cleavage reporter where upon $\mathrm{HCV}$ NS3-4A cleavage of the reporter, the RFP translocates to the nucleus [29]. Translocation of RFP was observed with full-length RHV1, confirming the previous finding that the RHV1 NS3-4A protease is capable of cleaving human MAVS [30]; however, the number of cells with RFP translocation did not increase over time (Figure S3.A).

We further tested full-length viral constructs containing $\mathrm{m}$-Scarlet and BSD inserted between NS4A-B in both Hepa1-6 and BHK-21, mScarlet was expressed in cells but the number of cells expressing mScarlet did not increase overtime and failed to yield a clone when selecting for replication with $\mathrm{BSD}$, even when expressing Sec14L2 and ApoE, both essential for high levels of $\mathrm{HCV}$ replication [31, 32] (Figure S3.B). Further cell lines could be tested along with knocking out the innate immune response in future experiments.
In Summary, this study shows that RHV1/2 and RPgV contain IRESs that are capable of driving high levels of protein synthesis. RHV1 structural genes are cleaved by cellular proteases and can be used to pseudotype lentivirus vectors that are capable of transducing murine hepatocytes.

\section{Data Availability}

The data used to support the findings of this study are available from the corresponding author upon request.

\section{Disclosure}

This paper was previously made available in preprint on bioRxiv (doi: https://doi.org/10.1101/761379).

\section{Conflicts of Interest}

The authors declare that they have no conflicts of interest.

\section{Authors' Contributions}

SS designed and planned all the experiments. SS, SB, and KM performed the experiments and analysed results. SS and SB wrote the manuscript. CF edited the manuscript and acquired funding. 


\section{Supplementary Materials}

(1) Illustration of plasmid construction as outlined in methods. (2) Supplementary analyses of level of RNA transcripts produced by viral 5' UTRs. (3) MAVS cleavage and dsRNA analysis of full-length replicons. (Supplementary Materials)

\section{References}

[1] World Health Organization, Global Hepatitis Report, World Health Organization, Jeneva, Switzerland, 2017.

[2] C. Hézode, "Treatment of hepatitis C: results in real life," Liver International, vol. 38, no. S1, pp. 21-27, 2018.

[3] G. V. Papatheodoridis, A. Hatzakis, E. Cholongitas et al., "Hepatitis C: the beginning of the end-key elements for successful European and national strategies to eliminate HCV in Europe," Journal of Viral Hepatitis, vol. 25, pp. 6-17, 2018.

[4] H. J. Alter, "Discovery of the non-A, non-B hepatitis virus: the end of the beginning or the beginning of the end," Transfusion Medicine Reviews, vol. 3, pp. 77-81, 1989.

[5] A. Kapoor, P. Simmonds, G. Gerold et al., "Characterization of a canine homolog of hepatitis $\mathrm{C}$ virus," Proceedings of the National Academy of Sciences of the United States of America, vol. 108, pp. 11608-11613, 2011.

[6] J. F. Drexler, V. M. Corman, M. A. Müller et al., "Evidence for novel hepaciviruses in rodents," PLoS Pathogens, vol. 9, Article ID e1003438, 2013.

[7] A. Kapoor, P. Simmonds, T. K. H. Scheel et al., "Identification of rodent homologs of hepatitis C virus and pegiviruses," MBio, vol. 4, pp. e00216-e00313, 2013.

[8] S. Lyons, A. Kapoor, C. Sharp et al., "Nonprimate hepaciviruses in domestic horses, United Kingdom," Emerging Infectious Diseases, vol. 18, pp. 1976-1982, 2012.

[9] P. D. Burbelo, E. J. Dubovi, P. Simmonds et al., "Serologyenabled discovery of genetically diverse hepaciviruses in a new host," Journal of Virology, vol. 86, pp. 6171-6178, 2012.

[10] M. Lauck, S. D. Sibley, J. Lara et al., "A novel hepacivirus with an unusually long and intrinsically disordered NS5A protein in a wild old world primate," Journal of Virology, vol. 87, pp. 8971-8981, 2013.

[11] C. Baechlein, N. Fischer, A. Grundhoff et al., "Identification of a novel hepacivirus in domestic cattle from Germany," Journal of Virology, vol. 89, pp. 7007-7015, 2015.

[12] V. M. Corman, A. Grundhoff, C. Baechlein et al., "Highly divergent hepaciviruses from African cattle," Journal of Virology, vol. 89, pp. 5876-5882, 2015.

[13] M. Shi, X.-D. Lin, N. Vasilakis et al., "Divergent viruses discovered in arthropods and vertebrates revise the evolutionary history of the flaviviridae and related viruses," Journal of Virology, vol. 90, pp. 659-669, 2016.

[14] C. Firth, M. Bhat, M. A. Firth et al., "Detection of zoonotic pathogens and characterization of novel viruses carried by commensal Rattus norvegicus in New York city," MBio, vol. 5, pp. e01933-e02014, 2014.

[15] S. Trivedi, S. Murthy, H. Sharma et al., "Viral persistence, liver disease, and host response in a hepatitis C-like virus rat model," Hepatology, vol. 68, pp. 435-448, 2018.

[16] E. Billerbeck, R. Wolfisberg, U. Fahnøe et al., "Mouse models of acute and chronic hepacivirus infection," Science, vol. 357, pp. 204-208, 2017.

[17] O. G. Pybus and J. Thézé, "Hepacivirus cross-species transmission and the origins of the hepatitis C virus," Current Opinion in Virology, vol. 16, pp. 1-7, 2016.
[18] S. Pfaender, R. J. P. Brown, T. Pietschmann, and E. Steinmann, "Natural reservoirs for homologs of hepatitis C virus," Emerging Microbes and Infections, vol. 3, no. 3, e21 pages, 2014.

[19] C. L. Jopling, M. Yi, A. M. Lancaster, S. M. Lemon, and P. Sarnow, "Modulation of hepatitis $\mathrm{C}$ virus RNA abundance by a liver-specific MicroRNA," Science, vol. 309, pp. 15771581, 2005.

[20] E. L. Mohr and J. T. Stapleton, "GB virus type C interactions with HIV: the role of envelope glycoproteins," Journal of Viral Hepatitis, vol. 16, pp. 757-768, 2009.

[21] H. L. Tillmann and M. P. Manns, "GB virus-C infection in patients infected with the human immunodeficiency virus," Antiviral Research, vol. 52, pp. 83-90, 2001.

[22] M. Lauck, A. L. Bailey, K. G. Andersen, T. L. Goldberg, P. C. Sabeti, and D. H. O'Connor, "GB virus C coinfections in west african ebola patients," Journal of Virology, vol. 89, pp. 2425-2429, 2015.

[23] S. Chandriani, P. Skewes-Cox, W. Zhong et al., "Identification of a previously undescribed divergent virus from the flaviviridae family in an outbreak of equine serum hepatitis," Proceedings of the National Academy of Sciences of the United States of America, vol. 110, pp. E1407-E1415, 2013.

[24] A. Kapoor, P. Simmonds, J. M. Cullen et al., "Identification of a pegivirus (GB virus-like virus) that infects horses," Journal of Virology, vol. 87, pp. 7185-7190, 2013.

[25] T. Masaki, R. Suzuki, M. Saeed et al., "Production of infectious hepatitis C virus by using RNA polymerase I-mediated transcription," Journal of Virology, vol. 84, pp. 5824-5835, 2010.

[26] J.-K. Oem, Z. Xiang, Y. Zhou, L. A. Babiuk, and Q. Liu, "Utilization of RNA polymerase I promoter and terminator sequences to develop a DNA transfection system for the study of hepatitis C virus internal ribosomal entry site-dependent translation," Journal of Clinical Virology, vol. 40, pp. 55-59, 2007.

[27] M. Flint, J. M. Thomas, C. M. Maidens et al., "Functional analysis of cell surface-expressed hepatitis C virus E2 glycoprotein," Journal of Virology, vol. 73, pp. 6782-6790, 1999.

[28] J. E. Reynolds, A. Kaminski, H. J. Kettinen et al., "Unique features of internal initiation of hepatitis C virus RNA translation," The EMBO Journal, vol. 14, pp. 6010-6020, 1995.

[29] C. T. Jones, M. T. Catanese, L. M. J. Law et al., "Real-time imaging of hepatitis $\mathrm{C}$ virus infection using a fluorescent cellbased reporter system," Nature Biotechnology, vol. 28, pp. 167-171, 2010.

[30] Anggakusuma, R. J. P. Brown, D. H. Banda et al., "Hepacivirus NS3/4A proteases interfere with MAVS signaling in both their cognate animal hosts and humans: implications for zoonotic transmission," Journal of Virology, vol. 90, no. 23, pp. 10670-10681, 2016.

[31] J. Jiang, W. Cun, X. Wu, Q. Shi, H. Tang, and G. Luo, "Hepatitis $\mathrm{C}$ virus attachment mediated by apolipoprotein $\mathrm{E}$ binding to cell surface heparan sulfate downloaded from," Journal of Virology, vol. 86, pp. 7256-7267, 2012.

[32] M. Saeed, U. Andreo, H.-Y. Chung et al., "SEC14L2 enables pan-genotype $\mathrm{HCV}$ replication in cell culture," Nature, vol. 524, pp. 471-475, 2015. 